\title{
Treating malignant glioma in Chinese patients: update on temozolomide
}

This article was published in the following Dove Press journal:

OncoTargets and Therapy

12 February 2014

Number of times this article has been viewed

\author{
Liang Chang' \\ Jun $\mathrm{Su}^{\prime}$ \\ Xiuzhi Jia ${ }^{2,3}$ \\ Huan Ren ${ }^{2,3}$ \\ 'Department of Neurosurgery, \\ The Tumor Hospital of Harbin \\ Medical University, ${ }^{2}$ Department \\ of Immunology, Harbin Medical \\ University, ${ }^{3}$ Key Lab Infection and \\ Immunity, Heilongjiang Province, \\ Harbin, People's Republic of China
}

Correspondence: Huan Ren

Key Lab Infection and Immunity, Heilongjiang Province, Harbin I50086,

People's Republic of China

$\mathrm{Tel}+8645$ I 86674566

Fax +8645 I 86697322

Email renhuan@ems.hrbmu.edu.cn
Abstract: Malignant glioma, ie, anaplastic astrocytoma and glioblastoma, is the most common type of primary malignant brain tumor in the People's Republic of China, and is particularly aggressive. The median survival of patients with newly diagnosed glioblastoma is only 12-14 months despite advanced therapeutic strategies. Treatment of malignant glioma consists mainly of surgical resection followed by adjuvant radiation and chemotherapy. Temozolomide (TMZ), a second-generation oral alkylating agent, is playing an increasingly important role in the treatment of malignant glioma in Chinese patients. Since the publication of a study by Stupp et al in 2005, which used a protocol of conventional fractionated irradiation with concomitant TMZ followed by standard TMZ for six cycles, many clinical studies in the People's Republic of China have demonstrated that such a treatment strategy has significantly improved efficacy with limited side effects for newly diagnosed glioblastoma after surgery as compared with strategies that do not contain TMZ. However, as a relatively new agent, the history and development of TMZ for malignant glioma is not well documented in Chinese patients. Multicenter, randomized controlled trials including appropriately sized patient populations investigating multiple aspects of TMZ therapy and related combination therapies are warranted in patients with malignant glioma. This review provides an update on the efficacy, mechanism of action, adverse reactions, and clinical role of TMZ in the treatment of malignant glioma in Chinese patients.

Keywords: malignant glioma, chemotherapy, temozolomide, efficacy, side effect, People's Republic of China

\section{Introduction}

Glioma, which affects glial cells, is the most common primary brain tumor. The 2007 World Health Organization classification of central nervous system tumors separates glioma into grades I-IV, whereby grade I and II are defined as low grade and grade III and IV as high grade (also known as malignant glioma). Malignant glioma includes anaplastic glioma (anaplastic oligodendroglioma, anaplastic astrocytoma, and anaplastic oligoastrocytoma) and glioblastoma. The incidence of the tumor in the People's Republic of China is $1-4 / 100,000 ;{ }^{1}$ it may develop at any age, and has a peak incidence in the fifth and sixth decades of life. ${ }^{1}$ Malignant glioma generally presents with headache, cognitive change, epilepsy, dysphasia, and/or progressive hemiparesis, indicating destruction of normal brain tissue and widespread tumor invasion. Invasive tumor behavior is usually characterized by edema and contrast enhancement on computed tomography or magnetic resonance neuroimaging. Even though the clinical and neuroimaging features may be highly suggestive, additional histologic examination is accepted as the gold standard for diagnosis. ${ }^{2}$ The median survival for patients with grade III glioma, ie, anaplastic 
oligodendroglioma, anaplastic astrocytoma, or anaplastic oligoastrocytoma, is around 3 years. ${ }^{1}$ This type of tumor grows more rapidly than lower grade tumors, tends to invade nearby tissue, and shows histologic features of increased cellularity, nuclear atypia, and marked mitotic activity. In comparison, grade IV glioblastoma, the most severe type of brain tumor, is histologically defined by features of vascular proliferation, hypercellularity, pleomorphism, and pseudopalisading necrosis..$^{2-4}$ Glioblastoma accounts for $12 \%-15 \%$ of all intracranial tumors and $50 \%-60 \%$ of astrocytic tumors. Based on their clinical characteristics, glioblastomas are divided into primary and secondary tumors. The majority of cases $(>90 \%)$ are primary glioblastoma, arise as a de novo process, and mainly affect the elderly. Secondary glioblastoma develops progressively from low grade astrocytoma and is more common in younger patients (mean age 45 years versus 62 years). ${ }^{5}$

The recent development of a molecular classification for malignant glioma has been helpful for the diagnosis and treatment of glioma, and is complementary to the current classification which is based mainly on histopathology. For example, it demonstrates that loss of $1 \mathrm{p} / 19 \mathrm{q}$ heterozygosity is a prognostic factor in patients with anaplastic oligodendroglioma, and such tumors are sensitive to chemotherapeutic agents and radiotherapy. ${ }^{6}$ Genetic alterations, such as amplification of EGFR and mutations of TP53, are markers suggestive of primary and secondary glioblastoma. Over the last decade, several diagnostic and prognostic biomarkers for malignant glioma have been reported; these efforts significantly impact current standards regarding diagnosis, therapeutic decisionmaking, and the prognosis for patients.

The treatment strategies presently available for malignant glioma include removing the local lesion by surgery followed by adjuvant radiation and chemotherapy. ${ }^{6,7}$ Despite considerable efforts, however, the prognosis for these patients is still poor in view of high recurrent rates. Resistance to radiation and chemotherapy is the main problem in the treatment of malignant glioma and reflects multiple mechanisms, including molecular resistance to DNA damage and apoptosis, attenuation of cytotoxicity by the microenvironment, and our limited ability to deliver drugs into the brain via the blood-brain barrier. Nonetheless, chemotherapy is playing an increasingly important role in the treatment options. Currently, concomitant radiochemotherapy with temozolomide (TMZ), an alkylating agent, is used widely in the People's Republic of China, and has been shown to reduce the risk of recurrence and prolong patient survival. ${ }^{6,8}$ However, being a relatively new agent, the history and development of
TMZ chemotherapy for malignant glioma in Chinese patients is not well documented. This review focuses on the efficacy, mechanisms of action, adverse reactions, and clinical application of TMZ in the treatment of malignant glioma in the People's Republic of China.

\section{Management of malignant glioma in Chinese patients}

As in other parts of the world, standard treatment for malignant glioma in Chinese patients includes surgery followed by radiotherapy and chemotherapy. Maximal tumor resection plays an important role in the treatment of this tumor. ${ }^{9}$ Retrospective studies in the past suggest that aggressive surgical resection is associated with longer survival. ${ }^{6}$ Recent technological advances in neurosurgery, including preoperative and intraoperative magnetic resonance imaging (MRI), functional mapping, sonographically guided cerebral glioma surgery, and the neuronavigation system have brought about meaningful improvements in the safety of resection and clinical benefits for patients with glioblastoma. ${ }^{9-11}$ A recent randomized, prospective, triple-blind, single-center, parallel-group, controlled clinical trial assessed the effect of intraoperative MRI-guided surgery in 142 glioma patients. ${ }^{12}$ Complete resection rates in the $3.0 \mathrm{~T}$ intraoperative MRI and control groups were $88.24 \%$ and $66.67 \%$, respectively $(P=0.1596)$. Nonetheless, these data provided high-level evidence for application of this new technology in surgery for Chinese patients with high grade glioma.

Because of their typically aggressive growth, malignant glioma cells are almost impossible to remove completely. ${ }^{9}$ Radiotherapy and chemotherapy are thus necessary following surgery to improve the overall efficacy of treatment. Radiotherapy, which is usually started within 4 weeks of surgery, is an integral part of treatment in the People's Republic of China. ${ }^{13}$ Conventional external irradiation (total dose 50-60 Gy 25-30 times for a period of 5-6 weeks), threedimensional conformal radiotherapy (total dose 60 Gy, 2-3 Gy per session, once daily, and 5 times per week), and conformal intensity-modulated radiotherapy (20 Gy/4 fractions/2 weeks) are often used; ${ }^{14,15}$ the latter two methods have the advantage in terms of a homogeneous dose distribution, precise targeting, and causing less damage to normal surrounding tissue. ${ }^{16}$

Chemotherapy first became available for glioma in the 1950s, but relevant clinical studies did not start to be reported in the People's Republic of China until the early 1970s. ${ }^{17}$ The traditional chemotherapeutic agents consist of small-molecule drugs that are highly lipid-soluble and able 
to penetrate the blood-brain barrier. ${ }^{18}$ These agents include carmustine, lomustine, nimustine, and semustine (Table 1). However, use of these agents achieved only modest improvement in progression-free survival and overall survival in patients with malignant glioma. ${ }^{19}$ These alkylating agents are also highly toxic to the gastrointestinal tract and bone marrow, which limits their clinical application. In comparison, TMZ, a newer orally administered alkylating agent with significantly improved efficacy and safety, has become firstline chemotherapy for patients with malignant glioma in the People's Republic of China.

Clinical studies demonstrate that radiotherapy and TMZ used in combination are superior to either treatment alone. ${ }^{6,14,15}$ However, before the advent of TMZ, irradiation was followed by chemotherapy with nimustine (or carmustine/lomustine), alone or in combination with other agents for newly diagnosed patients after surgery. Combinations including VM-26 (teniposide), a topoisomerase II inhibitor, or a platinumcontaining drug such as cisplatin were often used. ${ }^{18}$ These regimens are still commonly used as chemotherapy in the People's Republic of China (Table 1) because TMZ remains relatively expensive.

An increasing number of Chinese patients with malignant glioma are enjoying longer survival with postsurgical treatment based on the Stupp protocol published in 2005..$^{17,20}$ This protocol includes a total radiation dose of 55-60 Gy and TMZ $75 \mathrm{mg} / \mathrm{m}^{2} /$ day from 1 to 42 days during irradiation, followed by six cycles ofTMZ $150-200 \mathrm{mg} / \mathrm{m}^{2}$ on days $1-5$, repeated on day $29 .{ }^{21}$ This regimen is recommended for patients with newly diagnosed malignant glioma in the 2012 Chinese guideline for diagnosis and treatment of central nervous system tumors. ${ }^{1}$ Mao et al studied the efficacy of a modified version of this regimen, ie, early additional treatment with TMZ $\left(75 \mathrm{mg} / \mathrm{m}^{2}\right.$ on postoperative days 15-28) before radiation. One hundred and two patients with this malignant tumor were enrolled postoperatively and randomly divided into an early TMZ group and a Stupp group. Median survival and overall survival were longer in the early TMZ group than in the Stupp group (19.0 months versus 14.6 months and 17.6 versus 13.2 months, respectively), but the side effects were similar. ${ }^{22}$ Currently, the number of studies of TMZ chemotherapy accounts for $40 \%$ (Figure 1) and this proportion is increasing in the People's Republic of China, ${ }^{18}$ with doctors actively investigating the optimized TMZ strategies most suitable for their patients.

The majority of malignant glioma recurs locally. ${ }^{23}$ Repeat resection in patients with recurrent disease should take a number of aspects into account, and be undertaken in younger patients, those with a Karnovsky performance status $>70$ and a suitable interval between the first and second operation, and when the location of the lesion is favorable. ${ }^{24}$ Repeat irradiation remains controversial, whereas stereotactic radiosurgery or fractionated stereotactic radiosurgery with repeat focal radiation has been shown to be a useful adjunct in the treatment of recurrent glioblastoma. ${ }^{6}$

The optimal chemotherapy regimens for recurrent glioblastoma are not defined. Currently, combination therapies including TMZ and targeted agents, ie, small-molecule kinase inhibitors or antibodies, or switching to nonconventional TMZ regimens including dose-dense or metronomic regimens (refer to the following sections), are being tested for treatment of recurrent tumors. ${ }^{25,26}$ However, thus far, there is no relevant report concerning the use of TMZ in the treatment of Chinese patients with recurrent tumors. Moreover, elderly patients (age $>70$ years) constitute around half of the patients

Table I Overview of different chemotherapeutic agents and cost of treatment for a patient with malignant glioma*

\begin{tabular}{|c|c|c|c|c|c|c|c|c|c|}
\hline \multirow[t]{2}{*}{ Variable } & \multicolumn{3}{|c|}{ Nitrosoureas } & \multirow[t]{2}{*}{ VM-26 } & \multirow{2}{*}{$\begin{array}{l}\text { ACNU + } \\
\text { VM-26 }\end{array}$} & \multicolumn{2}{|c|}{ TMZ (Chinese) } & \multirow{2}{*}{$\begin{array}{l}\text { TMZ } \\
\text { (Western) }\end{array}$} & \multirow[t]{2}{*}{ Bevacizumab } \\
\hline & ACNU & BCNU & $\overline{M e C C N U}$ & & & $\overline{\text { Temadar }^{\circledR}}$ & Diyi ${ }^{\circledR}$ & & \\
\hline $\begin{array}{l}\text { Mode of } \\
\text { action }\end{array}$ & \multicolumn{3}{|c|}{ DNA chloroethylation } & $\begin{array}{l}\text { Topoisomerase } \\
\text { II inhibitor }\end{array}$ & $\begin{array}{l}\text { Combined } \\
\text { actions }\end{array}$ & \multicolumn{2}{|c|}{ DNA methylation } & & $\begin{array}{l}\text { Angiogenesis } \\
\text { inhibitor }\end{array}$ \\
\hline $\begin{array}{l}\text { BBB } \\
\text { penetration }\end{array}$ & + & + & + & + & + & ++ & ++ & ++ & - \\
\hline $\begin{array}{l}\text { Oral or } \\
\text { intravenous }\end{array}$ & Intravenous & Intravenous & Oral & Intravenous & Intravenous & Oral & Oral & Oral & Intravenous \\
\hline Cost $(\$)^{\wedge}$ & $61 / 25 \mathrm{mg}$ & $21 / 125 \mathrm{mg}$ & $3 / 50 \mathrm{mg}$ & $23 / 50 \mathrm{mg}$ & $\begin{array}{l}61 / 25 \mathrm{mg}+ \\
23 / 50 \mathrm{mg}\end{array}$ & $\begin{array}{l}41 / 20 \mathrm{mg} \\
143 / 100 \mathrm{mg}\end{array}$ & $52 / 50 \mathrm{mg}$ & $\begin{array}{l}35 / 20 \mathrm{mg} \\
102 / 100 \mathrm{mg}\end{array}$ & $853 / 100 \mathrm{mg}$ \\
\hline $\begin{array}{l}\text { Dose } \\
\left(\mathrm{mg} / \mathrm{m}^{2} / \text { day }\right)\end{array}$ & 90 & 100 & 150 & 60 & $90+60$ & $\begin{array}{l}75^{\dagger} \\
150-200^{\ddagger}\end{array}$ & $\begin{array}{l}75^{\dagger} \\
150-200^{\ddagger}\end{array}$ & $\begin{array}{l}75^{\dagger} \\
150-200^{\ddagger}\end{array}$ & $5-10 \mathrm{mg} / \mathrm{kg}$ \\
\hline $\begin{array}{l}\text { Total }(\$) / \\
6 \text { cycles }\end{array}$ & 2,182 & 369 & 3,060 & 8,316 & 10,498 & 25,444 & 17,050 & 19,130 & $15,327-30,629$ \\
\hline
\end{tabular}

Notes: *Data refer to acquisition cost only and were calculated for a patient with a $1.72 \mathrm{~m}^{2}$ body surface area; ${ }^{\wedge}$ price per unit drug is taken from the price list of the local hospital, according to the current exchange rate, I\$ being equal to $6.10 ¥ ;{ }^{\dagger}{ }^{\dagger}$ concomitant with irradiation regimen; ${ }^{\ddagger}$ adjuvant to irradiation regimen. Abbreviations: BBB, blood-brain barrier; ACNU, nimustine; BCNU, carmustine; MeCCNU, semustine; VM-26, teniposide; TMZ, temozolomide. 
A

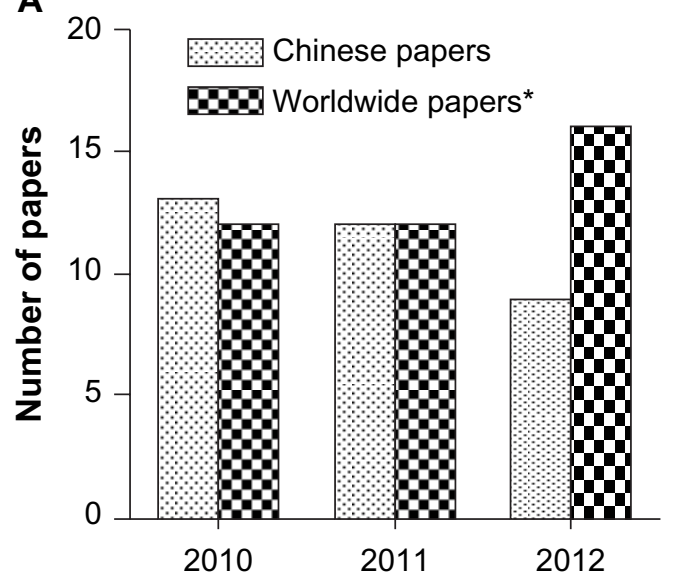

B

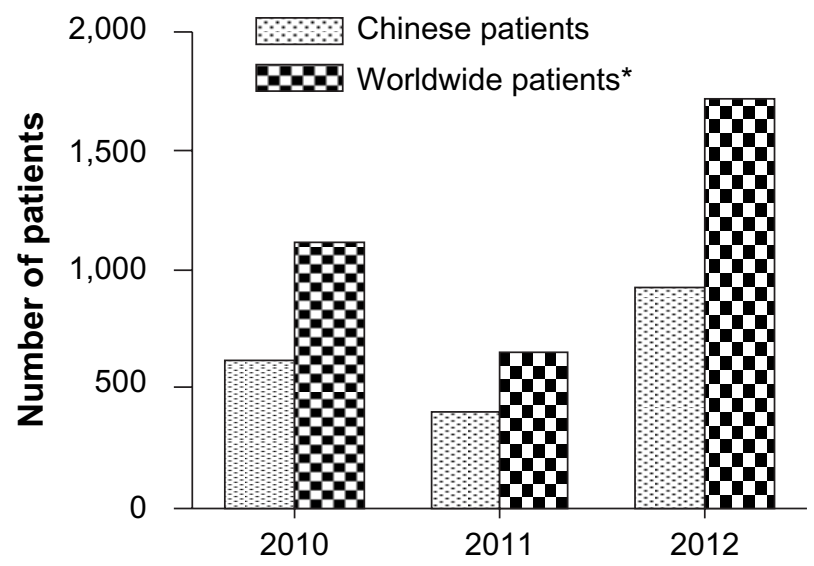

Figure I Comparison between Chinese and worldwide literature on use of temozolomide in high grade glioma. (A) Studies published in Chinese databases and PubMed from 2010 to 20I2. (B) Patients enrolled in studies identified in Chinese databases and PubMed from 2010 to 20I2. *The online PubMed database (http://www.ncbi.nlm.nih.gov) was considered representative of countries other than the People's Republic of China; the China Knowledge Resource Integrated Database and Chinese Medical Association Digital Periodicals were considered representative of the People's Republic of China. The databases were searched using the search terms "glioma", "glioblastoma", and "temozolomide" from January 2010 to October 2012. In the event that one study was the subject of several publications, the publication with the largest sample size was used. Inclusion criteria were: clinical study of temozolomide in high grade glioma and retrospective or prospective study design. Chinese studies were excluded from data obtained from the PubMed database. Forty papers including 3,470 patients were identified in PubMed and 34 papers including I,966 patients were identified in the Chinese databases.

with this type of malignant tumor, and recent data suggest that these patients benefit from active antitumor therapy in terms of palliation and prolonged survival. ${ }^{26} \mathrm{~A}$ short-course of hypofractionated radiotherapy is a reasonable alternative to standard radiotherapy. ${ }^{27} \mathrm{TMZ}$ should be used with caution in elderly patients with an unfavorable Karnovsky performance status $(<70)$. It has been reported that standard radiotherapy with concomitant TMZ may be an advantageous treatment option for elderly patients with newly diagnosed glioblastoma and good prognostic factors. ${ }^{27}$ Randomized, controlled studies in elderly patients are needed in the future.

\section{Overview of the pharmacology of temozolomide}

TMZ is a second-generation alkylating agent derived from dacarbazine, ${ }^{18}$ and is marketed in the People's Republic of China as Temodar ${ }^{\circledR}$ (Schering Corporation, Union, NJ, USA) or Diyi ${ }^{\circledR}$ (Tasly Group Co, Ltd, Tianjin). Temodar was approved by both the US Food and Drug Administration and European Medicines Agency in 1999 for the treatment of recurrent anaplastic astrocytoma, and is currently used as a first-line chemotherapeutic agent for malignant glioma. Temodar became available in the People's Republic of China in 2008, and Diyi appeared on the Chinese market as a generic version of Temodar in 2004, and has demonstrated effects similar to those of Temodar in the clinical treatment of brain tumors.

TMZ distributes rapidly to all tissues and penetrates the blood-brain barrier well. The half-life of TMZ is approximately 1.8 hours, with peak serum concentrations reached within 1-2 hours. ${ }^{28}$ The mean plasma area under the curve for TMZ after oral administration is $22.6 \mu \mathrm{g} /$ hour $/ \mathrm{mL}$ after a dose of $150 \mathrm{mg} / \mathrm{m}^{2}$. TMZ does not require hepatic metabolism for activation, and spontaneously hydrolyzes to 5-(3-methyltriazen-1-yl)imidazole-4-carboxamide (MTIC) and acid metabolites at physiologic $\mathrm{pH} .{ }^{29}$ MTIC is degraded further from $O^{6}$-alkylguanine-DNA alkyltransferase to 4-amino-5-imidazole-carboxamide (AIC), which is an intermediate in purine and nucleic acid biosynthesis. ${ }^{18}$ Relative to the area under the curve for TMZ, exposure to MTIC and AIC is $2.4 \%$ and $23 \%$, respectively. ${ }^{28}$ The drug is excreted via the kidney and overall clearance is about $5.5 \mathrm{~L} /$ hour $/ \mathrm{m}^{2}$.

TMZ is a cell cycle nonspecific agent. DNA methylation and failure of mismatch repair play a major role in the cytotoxicity of this agent. TMZ can transfer a methyl group to DNA, the most common being $N^{7}$-methylguanine (70\%), followed by $O^{3}$-methyladenine $(9 \%)$ and $O^{6}$-methylguanine (5\%). The cytotoxicity of MTIC is thought to be due primarily to $O^{6}$-methylguanine, which triggers, for example, futile DNA mismatch repair and breaks in double-stranded DNA breaks. Aberrant repair of the methyl adduct in the DNA mismatch repair system leads to inhibition of replication in the daughter cells, thereby blocking the cell cycle and inducing apoptosis. ${ }^{30,31}$ There are a number of factors that influence the cellular response to TMZ, and among them, $O^{6}$-methylguanine DNA methyltransferase is one of the most important. Thus, cells deficient in $M G M T$ or with 
depleted $O^{6}$-methylguanine DNA methyltransferase activity show increased sensitivity to TMZ. ${ }^{32}$ A second key regulator of TMZ sensitivity is the mismatch repair itself. Cells lacking a mismatch repair function do not recognize and repair the $O^{6}$-methylguanine adducts produced by TMZ; whereas these cells suffer from an accumulation of mutations, they do not undergo TMZ-induced G2 arrest and are resistant to TMZ-induced cell death. ${ }^{33}$ Nevertheless, other factors that may affect TMZ-induced DNA damage and link the damage to downstream cell death pathways may also contribute to $\mathrm{TMZ}$ resistance in glioma cells, so further investigations are much needed.

\section{Comparative studies with current treatment options}

The earliest clinical trial of TMZ in the treatment of Chinese patients with malignant glioma was initiated in $2001,{ }^{34}$ and the results were reported in $2003 .{ }^{34}$ It is estimated that, before 2009 , less than $30 \%$ of Chinese patients with malignant glioma could afford treatment with TMZ (Table 1), whereas approximately $80 \%$ of those in the large urban areas, such as Beijing and Shanghai, and less than $50 \%$ in other areas of the country have been treated with TMZ since 2009, when TMZ became included in the national drug insurance list (unpublished data). Currently, the following TMZ dosing schedules are supported in the People's Republic of China: the Stupp protocol, concomitant with irradiation (days 1-42/49), with a total radiation dose of 55-60 Gy and TMZ $75 \mathrm{mg} / \mathrm{m}^{2} /$ day for $42-49$ days during irradiation, followed by six cycles of TMZ $200 \mathrm{mg} / \mathrm{m}^{2}$ on days $1-5$, repeated on day 29; a standard regimen (5/28) of $150-200 \mathrm{mg} / \mathrm{m}^{2}$ on days $1-5$, on a cycle of 28 days; a metronomic regimen (28/28) of TMZ $50 \mathrm{mg} / \mathrm{m}^{2}$ continuously on days $1-28$, on a cycle of 28 days; a prolonged regimen $(21 / 28)$ of $75-100 \mathrm{mg} / \mathrm{m}^{2}$ on days $1-21$, on a cycle of 28 days; a dose-dense regimen $(7 / 7)$ of $150 \mathrm{mg} / \mathrm{m}^{2}$ on days $1-7$, repeated on day 8 ; a one week on/one week off regimen (7/14) of TMZ $150 \mathrm{mg} / \mathrm{m}^{2}$ on days $1-7$, repeated on day 15 ; and a $42 / 70$ regimen of TMZ $75 \mathrm{mg} / \mathrm{m}^{2} /$ day on days $1-42$, on a cycle of 70 days. Among these, the first two protocols are mostly used in Chinese patients. An increasing number of multicenter, randomized, controlled studies of TMZ chemotherapy are presently being carried out in the People's Republic of China. These studies already demonstrate that TMZ is not only a drug with reasonable efficacy and limited side effects for the treatment of malignant glioma, but also that it is an agent that may potentially enhance the antitumor activity of other therapeutic modalities, including radiotherapy and/or targeted agents used in combination therapies.

\section{TMZ versus traditional chemotherapy agents}

Compared with traditional chemotherapeutic agents, the effects of TMZ in malignant glioma have been remarkable; it has been reported that complete response and partial response rates are approximately $30 \%$ with $\mathrm{TMZ}$ versus only $10 \%$ with traditional chemotherapeutic agents..$^{30,35-37}$ The 2-year survival rate is improved from $10.4 \%$ to $26.5 \%$ with the use of TMZ. ${ }^{17,30,35,36}$ Qian et $\mathrm{al}^{35}$ compared the efficacy of TMZ and lomustine in patients with malignant glioma. Ninety-seven patients were randomly divided following surgery into a TMZ group and a lomustine group. The response rate in the TMZ and lomustine groups was $35.7 \%$ and $9.1 \%$, respectively, and the clinical benefit rate (complete response + partial response + stable disease) was $90.5 \%$ and $75.0 \%$. Although the therapeutic efficacy of TMZ in patients with glioblastoma was not optimal, it was better than that with traditional chemotherapeutic agents.

Another study investigated the clinical efficacy of TMZ versus nimustine-based chemotherapy in patients with newly diagnosed glioblastoma who had completed surgery and radiation therapy. ${ }^{19}$ Thirty-four patients received TMZ and 101 received nimustine-based chemotherapy. Median overall survival was significantly longer in the $\mathrm{TMZ}$ group than in the nimustine group $(P=0.011)$. In a multivariate Cox analysis adjusted for prognostic factors in all patients, treatment with $\mathrm{TMZ}$ independently predicted a favorable outcome ( $P=0.002$ for overall survival, hazard ratio 0.45 ; $P=0.011$ for progression-free survival, hazard ratio 0.56 ). Further, whereas most patients with malignant glioma experience disease recurrence, studies in the People's Republic of China indicate that single-agent TMZ therapy is effective to prolong survival for such tumors without previous treatment. Sun et $\mathrm{al}^{37}$ evaluated the efficacy of a standard regimen of TMZ with that of semustine $150 \mathrm{mg} / \mathrm{m}^{2}$ on day one repeated every 28 days for three cycles in the treatment of recurrent glioma. A total of 144 patients with recurrence of glioblastoma or anaplastic astrocytoma were enrolled in this study. Progression-free survival at 6 months was $78.9 \%$ in the TMZ group and $55.9 \%$ in the semustine group $(P<0.05)$. Overall survival rates at the end of the follow-up period were $96.9 \%$ and $97.3 \%$, respectively $(P>0.05)$. However, with regard to relapse after standard-dose TMZ therapy, the effect was not obvious and the options were limited. Yan et $\mathrm{al}^{25}$ evaluated the 
efficacy of TMZ in the treatment of recurrent glioma, but the curative effect from readministration of TMZ was modest. These investigators concluded that patients who were treated first with TMZ had significantly higher rates of response and 6-month progression-free survival than patients retreated with TMZ. Much of the relevant data generated in the People's Republic of China is similar to that for studies performed in other countries, ${ }^{38-40}$ and shows that the therapeutic effect of a standard regimen of TMZ is more effective than that of traditional chemotherapy regimens with regard to progression-free survival, overall survival, complete response rate, and clinical benefit rate in patients with malignant glioma.

\section{Stupp regimen versus other treatment options}

An increasing number of clinicians are investigating the effect of treatment consisting of radiotherapy in combination with TMZ based on the Stupp protocol. ${ }^{21}$ As in Western countries, doctors in the People's Republic of China are taking advantage of this protocol or using modified methods to enhance the efficacy of treatment in patients with malignant glioma (Table 2). ${ }^{41-44}$ Based on this protocol, a prospective study was conducted by Zhao et al from 2006 to $2011^{45}$ in 287 patients with glioblastoma who were randomly divided postoperatively into a Stupp group $(\mathrm{n}=151)$ and a radiotherapy group $(n=136)$ in a proportion of 1:0.9. The mortality risk in the Stupp group compared with the radiotherapy group was 0.53 (95\% confidence interval $[\mathrm{CI}] 0.41-0.67 ; P<0.01$ ) adjusted according to age, sex, Mini-Mental State Examination, and Eastern Cooperative Oncology Group performance status. The extent of surgery was noted to have a significant influence on the prognosis. The median survival of patients in the Stupp group and the radiotherapy group was 14.8 months and 11.7 months, respectively, and progression-free survival was 6.8 and 4.9 months. These results have been confirmed by other investigators. ${ }^{46}$

Wang et $\mathrm{al}^{14}$ retrospectively analyzed the effect of the Stupp regimen using either radiotherapy or single-agent TMZ postoperatively in 78 patients with malignant glioma. The radiotherapy group received a total radiation dose of $60 \mathrm{~Gy}$ whereas the TMZ group received six cycles of TMZ 150-200 $\mathrm{mg} / \mathrm{m}^{2}$ on days $1-5$, repeated on day 29 , with both treatments initiated 2 weeks following surgery The 3-year survival rates were $20.8 \%, 20.0 \%$, and $41.4 \%$, and progression-free survival was $17.7,17.9$, and 23.3 months in the TMZ, radiotherapy, and TMZ + radiotherapy groups, respectively. Collectively, the results showed that use of TMZ according to the Stupp regimen was more effective following surgery in patients with malignant glioma, with an increase in median survival from 12.1 months to 14.6 months, and in the 2-year survival rate from $8 \%$ to $26 \%{ }^{41}$

In another prospective study that included 96 postoperative patients with grade III-IV cerebral glioma, Zhai et al ${ }^{15}$ compared the efficacy and side effects between two groups $(n=48)$, ie, a TMZ + radiotherapy group whereas the VM-RT group had radiotherapy with VM-26 plus semustine. The median survival time and one-year, 2-year, and 3-year survival rates for patients in $\mathrm{TMZ}+$ radiotherapy group were 28 months, $72.95 \%, 54.2 \%$, and $31.3 \%$, respectively, indicating significantly better efficacy than in the VM + radiotherapy group (16 months, 62.55, 33.3\%, and 16.7\%, respectively, $P<0.05)$. These investigators concluded that radiotherapy

Table 2 Therapeutic activity of temozolomide for high grade glioma in Western and Chinese patients

\begin{tabular}{|c|c|c|c|c|}
\hline References & Cases (n) & Research subjects & $\begin{array}{l}\text { Methods and results } \\
\text { (RR, OR, or HR, } 95 \% \mathrm{Cl} \text { ) }\end{array}$ & Database \\
\hline $\begin{array}{l}\text { Yin et al }{ }^{41} \\
(2003-2012)\end{array}$ & 993 & $\begin{array}{l}\text { Elderly with GBM } \\
(>65 \text { years })\end{array}$ & $\begin{array}{l}\text { TMZ versus } R T \\
\quad \text { Increased OS (HR 0.86, } \mathrm{Cl} 0.74-1.00)\end{array}$ & $\begin{array}{l}\text { PubMed, EMBASE, } \\
\text { Cochrane }\end{array}$ \\
\hline \multirow[t]{2}{*}{$\begin{array}{l}\text { Hart et al }{ }^{42} \\
(1999-2007)\end{array}$} & 683 & High-grade & $\begin{array}{l}\text { TMZ plus RT versus RT } \\
\text { Increased OS (HR 0.58, } \mathrm{Cl} 0.50-0.68) \\
\text { Increased PFS: (HR 0.56, Cl 0.48-0.67) }\end{array}$ & $\begin{array}{l}\text { CENTRAL, Medline, } \\
\text { EMBASE, SCI, PDQ, } \\
\text { Meta-Register of }\end{array}$ \\
\hline & 225 & Recurrent high-grade & $\begin{array}{l}\text { TMZ plus RT versus RT alone } \\
\text { Increased OS (HR 0.87, } \mathrm{Cl} 0.65-\mathrm{I} .16 \text { ) } \\
\text { Increased PFS (HR } 0.68, \mathrm{Cl} 0.5 \mathrm{I}-0.90)\end{array}$ & Controlled Trials \\
\hline $\begin{array}{l}\text { Liang et } \mathrm{al}^{43} \\
(2007-2010)\end{array}$ & $24 I$ & High-grade & $\begin{array}{l}\text { TMZ plus RT versus RT } \\
\text { Increased OS (RR I.87, Cl I.47-2.38) }\end{array}$ & $\begin{array}{l}\text { *CNKI, SinoMed, } \\
\text { VIP databases }\end{array}$ \\
\hline $\begin{array}{l}\text { Wang et al }{ }^{44} \\
(2007-2008)\end{array}$ & 438 & High-grade & $\begin{array}{l}\text { TMZ versus other alkylating agents } \\
\text { Increased } P R(O R \quad 3.70, C l \text { 2.37-5.79) }\end{array}$ & $\begin{array}{l}\text { CNKI, SinoMed, VIP } \\
\text { databases }\end{array}$ \\
\hline
\end{tabular}

Note: ${ }^{*} \mathrm{CNKI}$, SinoMed, and VIP database are Chinese databases.

Abbreviations: HR, hazard ratio; OR, odds ratio; RR, relative risk; $\mathrm{Cl}$, confidence interval; OS, overall survival; PR, partial response; PFS, progression-free survival; TMZ, temozolomide; GBM, glioblastoma; RT, radiation therapy; EMBASE, Excerpta Medica Database; SCI, Science Citation Index; PDQ, Physician Data Query; CENTRAL, Cochrane Central Register of Controlled Trials; CNKI, Chinese National Knowledge Infrastructure; VIP, Very Important Person. 
with concurrent TMZ chemotherapy is an effective regimen with mild toxicity in patients with malignant glioma.

Moreover, other studies in the People's Republic of China show that expression of $O^{6}$-methylguanine DNA methyltransferase influences the cytotoxicity of TMZ. Jin et $\mathrm{al}^{47}$ compared the effects of TMZ combined with intensitymodulated radiotherapy (IMRT + TMZ group) with those of IMRT alone in patients with malignant glioma and positive $O^{6}$-methylguanine DNA methyltransferase on immunochemistry. The treatment effect was observed to be similar between the two regimens.

\section{TMZ in combination with targeted or other agents}

Although TMZ + radiotherapy has proven to be effective in the treatment of malignant glioma, the 5-year survival rate is less than $10 \%,{ }^{48}$ so more optimal treatment strategies are clearly needed. Malignant glioma is characterized by aberrant activation of signaling pathways. ${ }^{49}$ Several growth factor receptors, including vascular endothelial growth factor receptor and epidermal growth factor receptor, are mutated, overexpressed, and/or amplified, leading to increased cell proliferation and survival. ${ }^{49,50}$ An increased understanding of the molecular pathways involved in signal transduction, angiogenesis, and cell growth has led to the development of a number of targeted agents, including small molecule inhibitors such as tyrosine kinase inhibitors, matrix metalloproteinase inhibitors, histone deacetylase inhibitors, and antibodies. ${ }^{51}$ Currently, several targeted agents for potential use in combination with TMZ are being tested in malignant glioma, including glioblastoma. Yang et $\mathrm{al}^{52}$ evaluated the efficacy of nimotuzumab, a humanized monoclonal antibody against the epidermal growth factor receptor, in combination with $\mathrm{TMZ}$ in patients with glioblastoma. The disease control rate (partial response + stable disease) was $64.3 \%$, median progression-free survival was 4 months (95\% CI 0.7-7.3), and 6-month progression-free survival was 30.6\%. A study of treatment using a combination of TMZ and bevacizumab, an angiogenesis inhibitor, and other therapeutic agents in recurrent glioblastoma is ongoing. ${ }^{53}$ However, thus far, there are no published data from relevant clinical trials in the People's Republic of China. Recently, Yang et $\mathrm{al}^{54}$ have reported the results of their Phase II trial of TMZ + interferon- $\beta$ in 30 patients with recurrent high-grade tumors that were progressive or recurrent after prior standard radiotherapy + TMZ. The patients received interferon- $\beta 3$ MU on days 1, 3, and 5, and TMZ $200 \mathrm{mg} / \mathrm{m}^{2}$ on days 2-5 for a 4-week cycle until tumor progression. The median progression-free survival was 10.0 months (95\% CI $0.5-19.5)$ versus 5.0 months (95\% CI 3.0-7.0) months, respectively, for patients with grade III-IV disease; median overall survival was not available for grade III patients and was 9.5 months $(95 \%$ CI 7.7-11.3) for grade IV patients $(P<0.05)$. The authors concluded that $\mathrm{TMZ}+$ interferon- $\beta$ had moderate activity for recurrent high-grade glioma with acceptable toxicity.

\section{Safety and tolerability issues}

Unlike the traditional alkylating agents, TMZ is a well tolerated drug. Its side effects are divided into four grades of toxicity according to the National Cancer Institute Common Toxicity Criteria version $2.0,{ }^{55}$ and include three categories, ie, myelosuppression, nonhematologic toxicity, and infection. ${ }^{56} \mathrm{TMZ}$ does not cross-link DNA strands, so the direct toxic effect regarding hematopoietic progenitor cells is small as compared with conventional alkylating agents. ${ }^{23,57,58} \mathrm{~A}$ large number of clinical trials show that bone marrow suppression caused by TMZ is mild to moderate in Chinese patients. ${ }^{20,59-61}$ Yao et al $^{20}$ observed side effects in 32 patients with malignant glioma treated by TMZ and reported that grade III-IV neutropenia was the most common toxicity in long-term treatment, occurring in $9.4 \%(3 / 32)$ of their patients. Moreover, this toxicity could disappear one week after administration of TMZ or resolve in 3-5 days with administration of granulocyte-colony stimulation factor, which stimulates the bone marrow to produce and release granulocytes and stem cells into the bloodstream. Zhou et a ${ }^{59}$ observed adverse effects in 123 patients with glioma (42 low grade and 81 high grade) treated with TMZ and reported hematologic toxicity in 16 cases $(13.0 \%)$. Other nonhematologic toxicities include fatigue, alopecia, and gastrointestinal symptoms. These manifestations were seen to improve significantly within one week of cessation of chemotherapy. ${ }^{20,62} \mathrm{~A}$ study by $\mathrm{Li}^{62}$ including 32 patients reported that the mild to moderate toxicities of $\mathrm{TMZ}$ were fatigue $(86.9 \%)$, neutropenia syndrome $(46.9 \%)$, alopecia (46.9\%), constipation (41.2\%), thrombocytopenia (40.6\%), vomiting (34.4\%), and lymphopenia (25.0\%). Although fatigue was common, patients' daily activities were not affected. A meta-analysis ${ }^{63}$ of 896 patients from five randomized controlled trials investigated the safety of radiotherapy + TMZ in the treatment of newly diagnosed glioblastoma in the People's Republic of China. There were no differences in the incidence of grade IV neutropenia (relative risk 2.8, 95\% CI 0.1-67.1), grade III-IV thrombocytopenia (relative risk 4.0, 95\% CI 0.7-23.3), or grade III-IV leukopenia (relative risk 4.3, 95\% CI 0.8-24.3) between radiotherapy + TMZ 
and radiotherapy alone. An increasing number of reports are confirming that TMZ has the advantages of good tolerance, fewer side effects, and marked efficacy. There are several clinical studies ${ }^{64-66}$ indicating that long-term use of TMZ may increase the risk of carcinogenesis and activate hepatitis B, but these findings need confirmation by further studies.

\section{Other issues concerning TMZ}

Many aspects of TMZ chemotherapy require further investigation in the People's Republic of China. Compared with other chemotherapeutic agents, TMZ is more expensive, accounting for an annual expenditure of one billion US dollars worldwide. ${ }^{67,68}$ Not all Chinese patients with malignant glioma can afford TMZ (Table 1). The People's Republic of China is a developing country, and reforms in the health care and medical insurance systems are ongoing. In order to make rational decisions regarding treatment, it is necessary for patients, clinicians, and policy-makers to undertake a local economic evaluation of TMZ therapy. A recent study by $\mathrm{Wu}$ et $\mathrm{al}^{69}$ investigated the 5-year direct medical costs and health outcomes for three types of therapy, including radiotherapy, nitrosourea agents + radiotherapy, and $\mathrm{TMZ}+$ radiotherapy for newly diagnosed glioblastoma. The data suggest that the gap between the costs of TMZ and the ability to pay in a health resource-limited setting was too great to allow TMZ to be endorsed as the most appropriate therapeutic approach (Table 1). However, with the addition of TMZ to the national insurance schedule, such kinds of cost-effective situation are now changing.

Another issue is health-related quality of life in patients receiving TMZ. Health-related quality of life is a relatively new medical concept in the People's Republic of China, and encompasses a number of aspects, including physical, emotional, cognitive, and social functioning, as well as spiritual well-being. TMZ has relatively low toxicity and fewer side effects, so is beneficial in terms of improving health-related quality of life. Currently, the Karnovsky performance status questionnaire is the only instrument used to evaluate health-related quality of life in Chinese patients with malignant glioma. Wang et $\mathrm{al}^{70}$ assessed the effect of TMZ on health-related quality of life in 86 patients, and showed that addition of TMZ to the standard treatment of surgery and radiotherapy had a positive impact. Their data were confirmed by Fu et $\mathrm{al}^{71}$ in a further 28 patients with the same type of tumor. However, merely using the Karnovsky Performance Status questionnaire had shortcomings; whereas it is sensitive to neurologic impairment, it does not canvass social or psychologic dysfunction. Thus, further studies using more relevant questionnaires are warranted. Among the issues concerning TMZ therapy in patients with malignant glioma are financial status, patient attitudes towards short-term versus long-term clinical outcome, and nursing considerations, and these should be evaluated in a formal way, along with well designed clinical trials investigating related medical issues.

\section{Conclusion}

Chemotherapy has now become the standard of care for malignant glioma in Chinese patients. As in other parts of the world, addition of TMZ chemotherapy to integrated surgery and radiation regimens has significantly improved the prognosis of newly diagnosed glioblastoma, with an increase in 5-year overall survival from $1.9 \%$ to $9.8 \%{ }^{24}$ Since its introduction in 2003, TMZ therapy has become first-line chemotherapy in the treatment of newly diagnosed malignant glioma. ${ }^{18}$ However, although different chemotherapeutic agents and regimens and various methods of drug delivery have been tested, the paucity of multicenter, randomized, controlled trials with high-quality results remains a major limitation. Nevertheless, TMZ is playing an increasingly important role in the integrated treatment options for malignant brain tumors in Chinese patients. Investigations of optimized treatment regimens using single-agent TMZ or drugs in combination are ongoing, and much effort and collaboration have been undertaken in the People's Republic of China to generate well designed, multicenter, randomized, controlled trials of chemotherapy, aiming to improve further the prognosis of patients with malignant glioma.

\section{Acknowledgment}

This research was supported by the Scientific and Technological Project of Heilongjiang Province of China (GC12C303-1) and the Major Program of National Natural Science Foundation of China (NSFC 91229112).

\section{Disclosure}

The authors report no conflicts of interest in this work.

\section{References}

1. Zhou LF, Wang RZ, Bao SD, et al. [Chinese guideline for diagnosis and treatment on central nervous system tumors]. Zhonghua Yi Xue Za Zhi. 2012;92:2309-2313. Chinese.

2. Huse JT, Holland EC. Targeting brain cancer: advances in the molecular pathology of malignant glioma and medulloblastoma. Nat Rev Cancer. 2010;10:319-331.

3. Yin L, Zhang L. Correlation between MRI findings and histological diagnosis of brainstem glioma. Can J Neurol Sci. 2013;40:348-354.

4. Di Stefano AL, Enciso-Mora V, Marie Y, et al. Association between glioma susceptibility loci and tumour pathology defines specific molecular etiologies. Neuro Oncol. 2013;15:542-547. 
5. Ohgaki $\mathrm{H}$, Kleihues P. Genetic alterations and signaling pathways in the evolution of gliomas. Cancer Sci. 2009;100:2235-2241.

6. Yang P, Wang Y, Peng X, et al. Management and survival rates in patients with glioma in China (2004-2010): a retrospective study from a single-institution. J Neurooncol. 2013;113:259-266.

7. Chen C, Xu T, Lu Y, et al. The efficacy of temozolomide for recurrent glioblastoma multiforme. Eur J Neurol. 2013;20:223-230.

8. Strik HM, Marosi C, Kaina B, et al. Temozolomide dosing regimens for glioma patients. Curr Neurol Neurosci Rep. 2012;12:286-293.

9. Wu JS, Zhang J, Zhuang DX, et al. Current status of cerebral glioma surgery in China. Chin Med J (Engl). 2011;124:2569-2577.

10. Qiu TM, Yao CJ, Wu JS, et al. Clinical experience of $3 \mathrm{~T}$ intraoperative magnetic resonance imaging integrated neurosurgical suite in Shanghai Huashan Hospital. Chin Med J (Engl). 2012;125:4328-4333.

11. Wang J, Liu X, Ba YM, et al. Effect of sonographically guided cerebral glioma surgery on survival time. J Ultrasound Med. 2012;31: $757-762$.

12. Wu JS, Gong X, Song Y, et al. 142 3.0T iMRI guided resection in cerebral glioma surgery: interim analysis of a prospective, randomized, tripleblind, parallel-controlled trial. Neurosurgery. 2013;60 Suppl:167.

13. Pichlmeier U, Bink A, Schackert G, et al. Resection and survival in glioblastoma multiforme: an RTOG recursive partitioning analysis of ALA study patients. Neuro Oncol. 2008;10:1025-1534.

14. Wang L, Tu Q, Zhou W, et al. [Efficacy and safety of 3-dimensional conformal radiotherapy combined with temozolomide for glioma]. Zhong Nan Da Xue Xue Bao Yi Xue Ban. 2011;36:1106-1110. Chinese.

15. Zhai X, Wang J, Zhang J, et al. [Comparison of two regimens of postoperative concurrent chemoradiotherapy in adult patients with grade III-IV cerebral gliomas]. Nan Fang Yi Ke Da Xие Xие Bao. 2012;32:255-257. Chinese.

16. Ma X, Lv Y, Liu J, et al. Survival analysis of 205 patients with glioblastoma multiforme: clinical characteristics, treatment and prognosis in China. J Clin Neurosci. 2009;16:1595-1608.

17. Wang ZG, Yang WD, Yang SY, et al. [A clinical effect of temozolomide in patients with malignant glioma]. Journal of Tianjin Medical University. 2008;14:148-151. Chinese.

18. Sai K, Yang QY, Shen D, et al. Chemotherapy for gliomas in mainland China: an overview. Oncol Lett. 2013,5:1448-1452.

19. Wang Y, Chen X, Zhang Z, et al. Comparison of the clinical efficacy of temozolomide (TMZ) versus nimustine (ACNU)-based chemotherapy in newly diagnosed glioblastoma. Neurosurg Rev. 2014,37: 73-78.

20. Yao CX, Zhang SP, Chen B, et al. [Clinical effect of intensity modulated radiation therapy combined with concomitant and adjuvant temozolomide in the treatment for malignant glioma]. China Cancer J. 2013;22: 238-240. Chinese.

21. Brandes AA, Bartolotti M, Franceschi E. Second surgery for recurrent glioblastoma: advantages and pitfalls. Expert Rev Anticancer Ther. 2013;13:583-587.

22. Zhou LF, Yao Y, Yang S et al. The Stupp regimen preceded by early post-surgery temozolomide versus the Stupp regimen alone in the treatment of patients with newly diagnosed glioblastoma multiforme (GBM). J Clin Oncol. 2013;31 no. 15. Abstract 2022.

23. Chakrabarti I, Cockburn M, Cozen W, Wang YP, Preston-Martin S. A population-based description of glioblastoma multiforme in Los Angeles County, 1974-1999. Cancer. 2005;104:2798-2806.

24. Stupp R, Mason WP, van den Bent MJ, et al. Radiotherapy plus concomitant and adjuvant temozolomide for glioblastoma. $N$ Engl J Med. 2005;352:987-996.

25. Yan YI, Tang WY, Deng ZX, et al. [Efficacy of temozolomide in treatment of recurrent glioma]. Cancer Research on Prevention and Treatment. 2007;17:1359-1364. Chinese.

26. Reardon DA, Vredenburgh JJ, Desjardins A, et al. Effect of CYP3Ainducing anti-epileptics on sorafenib exposure: results of a phase II study of sorafenib plus daily temozolomide in adults with recurrent glioblastoma. J Neurooncol. 2011;101:57-66.
27. Wang W. [The treatment of elder patient with glioblastoma: a case report]. People's Military Surgeon. 2008;6:369-370. Chinese.

28. Tianjin Tasly Group Co [homepage on the Internet]. China: association of temozolomide. Online Resources [updated August 16, 2012; cited August 9, 2013]. Available from: http://www.tasly.com/. Accessed August 29, 2013.

29. Hong WQ, Zheng G, Wang FS, et al. [Advances of temozolomide in the treatment of malignant gliomas]. Chinese Journal of Neuro-oncology. 2008;6:260-264. Chinese

30. Shen D, Yang QY, Chen ZP. [Advances in the chemotherapy of malignant gliomas with temozolomide]. Chinese Journal of Neuro-oncology. 2012;4:271-276. Chinese.

31. Wang X, Chen JX, Liu YH, et al. Mutant TP53 enhances the resistance of glioblastoma cells to temozolomide by up-regulating $\mathrm{O}(6)$-methylguanine DNA-methyltransferase. Neurol Sci. 2013;34:1421-1428.

32. Boulton S, Pemberton LC, Porteous JK, et al. Potentiation of temozolomide-induced cytotoxicity: a comparative study of the biological effects of poly(ADP-ribose) polymerase inhibitors. Br J Cancer. 1995;72:849-856.

33. Wu Z, Chan CL, Eastman A, Bresnick E. Expression of human O6-methylguanine-DNA methyltransferase in Chinese hamster ovary cells and restoration of cellular resistance to certain N-nitroso compounds. Mol Carcinog. 1991;4:482-488.

34. Zeng X, Yang S. Clinical observation in chemotherapy with temozolomide alone in postoperative malignant primary cerebral glioma. Mod J Neurol Neurosurg. 2003;3:270-273.

35. Qian ZZ, Wang HQ, Liu XM, et al. [A multicenter randomized controlled study of temozolomide in 97 patients with malignant brain glioma]. Zhonghua Yi Xue Za Zhi. 2009;89:2059-2062. Chinese.

36. Ji YM, Xin L, Yang GK, et al. [Comparison of clinical effect between temozolomide and nimustine in patients with malignant gliomas]. Chinese Journal of Cancer Prevention and Treatment. 2007;14: 1021-1022. Chinese.

37. Sun J, Yang XJ, Yang SY. [Multicenter randomized controlled study of temozolomide versus semustine in the treatment of recurrent malignant glioma]. Zhonghua Yi Xue Za Zhi. 2013;93:165-168. Chinese.

38. Glas M, Happold C, Rieger J, et al. Long-term survival of patients with glioblastoma treated with radiotherapy and lomustine plus temozolomide. J Clin Oncol. 2009;27:1257-1261.

39. Happold C, Roth P, Wick W, et al. ACNU-based chemotherapy for recurrent glioma in the temozolomide era. J Neurooncol. 2009;92: 45-48.

40. Wick W, Hartmann C, Engel C, et al. NOA-04 randomized phase III trial of sequential radiochemotherapy of anaplastic glioma with procarbazine, lomustine, and vincristine or temozolomide. J Clin Oncol. 2009;27:5874-5880.

41. Yin AA, Zhang LH, Cheng JX, et al. Radiotherapy plus concurrent or sequential temozolomide for glioblastoma in the elderly: a metaanalysis. PLoS One. 2013;8:e74242.

42. Hart MG, Grant R, Garside R, et al. Temozolomide for high grade glioma. Cochrane Database Syst Rev. 2008;4:CD007415.

43. Liang H, Dong SL. [Effectiveness and safety of postoperative radiotherapy plus temozolomide for treating brain malignant glioma in China: a metaanalysis]. Modern Oncology. 2010;18:2345-2348. Chinese.

44. Wang YW, Yin CL, Zhang ZY, et al. [Meta-analysis of temozolomide for high grade gliomas]. $J$ Shandong University. 2012;50:80-83. Chinese

45. Zhao HX, Gao LM, Feng W, et al. [Effect of temozolomide plus radiotherapy in the treatment of glioblastoma]. Hainan Medical Journal. 2012:23:1-4. Chinese.

46. Xiao PX, Lin K, Chao SH. [Radiotherapy concomitant with temozolomide for glioblastoma multiforme]. Chinese Journal of Neuro-oncology. 2005;4:290-295. Chinese.

47. Jin WW, Zhang HY, Wang YT, et al. [The clinical observation of temozolomide combined with intensity-modulated radiotherapy in the treatment of malignant glioma with positive MGMT]. J Bengbu Med Coll. 2013;38:56-58. Chinese. 
48. Stupp R, Hegi ME, Mason WP, et al. Effects of radiotherapy with concomitant and adjuvant temozolomide versus radiotherapy alone on survival in glioblastoma in a randomised phase III study: 5-year analysis of the EORTC-NCIC trial. Lancet Oncol. 2009;10:459-466.

49. Katoh M. Networking of WNT, FGF, Notch, BMP, and Hedgehog signaling pathways during carcinogenesis. Stem Cell Rev. 2007;3: 30-38.

50. Xie Q, Xi G, Gong Y, et al. Protease activated receptor-1 and brain edema formation in glioma models. Acta Neurochir Suppl. 2013;118: 191-194.

51. Hutchinson L. Targeted therapies: glioma - it's all in the site occupancy. Nat Rev Clin Oncol. 2012;9:308.

52. Yang QY, Shen D, Sai K, et al. [Nimotuzumab in combination with chemotherapy for patients with malignant gliomas]. Zhonghua Zhong Liu Za Zhi. 2011;33:232-235. Chinese.

53. Chen XH, Zhou YX, Sun CM, et al. Combined modality therapy of recurrent glioma. J Clin Neurosurg. 2012:9:88-90.

54. Yang QY, Guo CC, Sai K, et al. [Phase II trial of temozolomide plus interferon- $\beta$ in recurrent malignant glioma patients]. Chinese Journal of Neuro-oncology. 2012;10:234-239. Chinese.

55. Trotti A, Byhardt R, Stetz J, et al. Common toxicity criteria: version 2.0. An improved reference for grading the acute effects of cancer treatment: impact on radiotherapy. Int J Radiat Oncol Biol Phys. 2000;47:13-47.

56. Dario A, Tomei G. The safety of temozolomide in patients with malignant glioma. Curr Drug Saf. 2006;1:205-222.

57. Minniti G, Salvati M, Arcella A, et al. Correlation between O6-methylguanine-DNA methyltransferase and survival in elderly patients with glioblastoma treated with radiotherapy plus concomitant and adjuvant temozolomide. J Neurooncol. 2011;102: 311-316.

58. Gerstner ER, Eichler AF, Plotkin SR, et al. Phase I trial with biomarker studies of vatalanib (PTK787) in patients with newly diagnosed glioblastoma treated with enzyme inducing anti-epileptic drugs and standard radiation and temozolomide. J Neurooncol. 2011;103: 325-332.
59. Zhou BY, Mao Q, Wang P, et al. [The adverse effects analysis of temozolomide in glioma chemotherapy]. Chinese Journal of Neuro-oncology. 2012;10:14-18. Chinese.

60. Levati L, Ruffini F, Muzi A, et al. Placenta growth factor induces melanoma resistance to temozolomide through a mechanism that involves the activation of the transcription factor NF-kappaB. Int J Oncol. 2011;38:241-247.

61. Lai A, Tran A, Nghiemphu PL, et al. Phase II study of bevacizumab plus temozolomide during and after radiation therapy for patients with newly diagnosed glioblastoma multiforme. J Clin Oncol. 2011;29:142-148.

62. Li G, Yang QY, Guo Y, et al. [Extended use of temozolomide for glioma patients based on MGMT expression pattern: experience of 32 cases]. Chinese Journal of Neuro-oncology. 2012;10:158-165. Chinese.

63. Wang G, Xiao H, Guo LY, et al. [Radiotherapy combined with temozolomide treatment for glioblastoma multiforme: a meta-analysis]. Tumor. 2010;30:1056-1065. Chinese.

64. Geiger H, Schleimer D, Nattamai KJ, et al. Mutagenic potential of temozolomide in bone marrow cells in vivo. Blood. 2006;107:3010-3011.

65. Grewal J, Dellinger CA, Yung WK, et al. Fatal reactivation of hepatitis B with temozolomide. N Engl J Med. 2007;356:1591-1592.

66. Goldbecker A, Tryc AB, Raab P, et al. Hepatic encephalopathy after treatment with temozolomide. J Neurooncol. 2011;103:163-166.

67. Klepper B, Pauker D. Medicare's drug plan: huge price disparities for common cancer drugs. Community Oncol. 2006;3:753-5511.

68. Crott R. The economics of temozolomide in brain cancer. Expert Opin Pharmacother. 2007;8:1923-1929.

69. Wu B, Miao Y, Bai Y, et al. Subgroup economic analysis for glioblastoma in a health resource-limited setting. PLoS One. 2012;7:e34588.

70. Wang Q, Wang NY, Sheng HM, et al. [Clinical observation in temozolomide combined with radiotherapy in treatment of postoperative malignant glioma]. Chin J Cancer Prev Treat. 2008;15:843-845. Chinese.

71. Fu H, Wan LL, Yang L, et al. [Clinical study of temozolomide combined with radiotherapy in treatment of postoperative malignant glioma]. Chinese General Practice. 2011;14:1556-1558. Chinese.
OncoTargets and Therapy

\section{Publish your work in this journal}

OncoTargets and Therapy is an international, peer-reviewed, open access journal focusing on the pathological basis of all cancers, potential targets for therapy and treatment protocols employed to improve the management of cancer patients. The journal also focuses on the impact of management programs and new therapeutic agents and protocols on

\section{Dovepress}

patient perspectives such as quality of life, adherence and satisfaction The manuscript management system is completely online and includes a very quick and fair peer-review system, which is all easy to use. Visit http://www.dovepress.com/testimonials.php to read real quotes from published authors. 